\title{
Development of a Biocatalytic Process for the Production of C6-Aldehydes from Vegetable Oils by Soybean Lipoxygenase and Recombinant Hydroperoxide Lyase
}

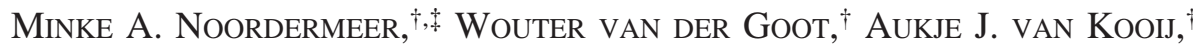 \\ Jan Willem VeldsinK, ${ }^{\S}$ Gerrit A. VeldinK, ${ }^{*}, \dagger$ AND \\ JOHANNES F. G. VLIEGENTHART ${ }^{\dagger}$ \\ Bijvoet Center for Biomolecular Research, Department of Bio-organic Chemistry, Padualaan 8, \\ 3584 CH Utrecht, The Netherlands; Agrotechnological Research Institute (ATO-DLO), \\ Wageningen, The Netherlands
}

\begin{abstract}
Volatile C6- and C9-aldehydes and alcohols are widely used as food flavors to reconstitute the "fresh green" odor of fruits and vegetables lost during processing. To meet the high demand for natural flavors, an efficient, cheap, and versatile biocatalytic process was developed to produce C6-aldehydes on a large scale. Vegetable oils were converted by soybean lipoxygenase and recombinant hydroperoxide lyase into hexanal and (2E)- or (3Z)-hexenal. In contrast to plant extracts, generally used as enzyme sources, high molar conversions were obtained with recombinant hydroperoxide lyase $(50 \%$ for hexanal and $26 \%$ for hexenal formation), and no side products were formed. Furthermore, recombinant hydroperoxide lyase lacks isomerase activity, allowing production of (3Z)hexenal, which could not be obtained in previously described processes. Recombinant hydroperoxide lyase is stable and can be stored at $4{ }^{\circ} \mathrm{C}$ for 1 month without significant loss of activity.
\end{abstract}

KEYWORDS: Lipoxygenase; hydroperoxide lyase; C6-aldehydes; biocatalysis; flavors

\section{INTRODUCTION}

Volatile C6- and C9-aldehydes and alcohols, such as hexanal, (3Z)- and (2E)-hexenal, (3Z)- and (2E)-nonenal, and (3Z,6Z)and $(2 E, 6 Z)$-nonadienal, are the main contributors to the characteristic odor of plants. They are widely used as food flavors to reconstitute the "fresh green" odor of fruits and vegetables, lost during processing. These compounds can be extracted from plants or synthesized. Chemical synthesis is not favored because consumers have a strong preference for natural food additives. Extraction is very expensive because of the low abundance of these short-chain aldehydes and alcohols in plants. Therefore, development of a biocatalytic process is required to produce these compounds on a large scale.

In plants, C6- and C9-aldehydes and alcohols are produced by the lipoxygenase pathway (Figure 1) and are involved in wound healing and pest resistance $(1,2)$. Linoleic (OD) and $\alpha$-linolenic acids (OT) are dioxygenated by lipoxygenase (LOX) to form 13- or 9-hydroperoxy-linole(n)ic acids (HPOD/T). The 13-hydroperoxy fatty acids can subsequently be cleaved by hydroperoxide lyase (HPL) into 12-oxo-(9Z)-dodecenoic acid and hexanal or (3Z)-hexenal, whereas the 9-hydroperoxy fatty acids can be cleaved into 9-oxononanoic acid and (3Z)-nonenal

* Author to whom correspondence should be addressed (e-mail g.a.veldink@chem.uu.nl; telephone +31 30 2532661; fax +31 30 2540980).

Bijvoet Center for Biomolecular Research.

$\doteqdot$ Present address: Shell Global Solutions, Amsterdam, The Netherlands.

$\S$ Agrotechnological Research Institute. or $(3 Z, 6 E)$-nonadienal. The (3Z)-aldehydes easily isomerize to their $(2 E)$-enal isomers and can be reduced to their corresponding alcohols by alcohol dehydrogenase.

Until now, plant extracts or cell cultures are the most common enzyme sources for biocatalytic production of C6- and C9aldehydes and alcohols. Large-scale conversion of fatty acids by lipoxygenase has successfully been accomplished (3-6). The subsequent conversion of hydroperoxy fatty acids by hydroperoxide lyase, however, caused problems. HPL in plant extracts is very unstable, and molar conversion rates were often $<1 \%$ $(7-10)$. Screening of different vegetables resulted in yields of $36 \%$ for hexanal and $24 \%$ for (2E)-hexenal (11). With hydroperoxide lyase from mung bean seedlings and linseed oil, molar conversions of $39 \%$ for $(2 E)$-hexenal and $21 \%$ for hexanal could be obtained (12). However, no (3Z)-hexenal was formed due to rapid isomerization to $(2 E)$-hexenal. Furthermore, the crude extracts contained other enzymes that used hydroperoxy fatty acids as substrates, such as allene oxide synthase and peroxygenase, leading to formation of undesired side products.

Recombinant expression would be an excellent method to increase the availability of hydroperoxide lyase for a biocatalytic process. The expression system we described before (13) has made it possible to obtain large quantities of stable hydroperoxide lyase with a high specificity for 13-hydroperoxylinoleic and $\alpha$-linolenic acids. Furthermore, the recombinant HPL forms (3Z)-hexenal, a compound that has been very difficult to produce because of its instability. Here, we describe the optimization of this expression system. The optimal reaction conditions of the 

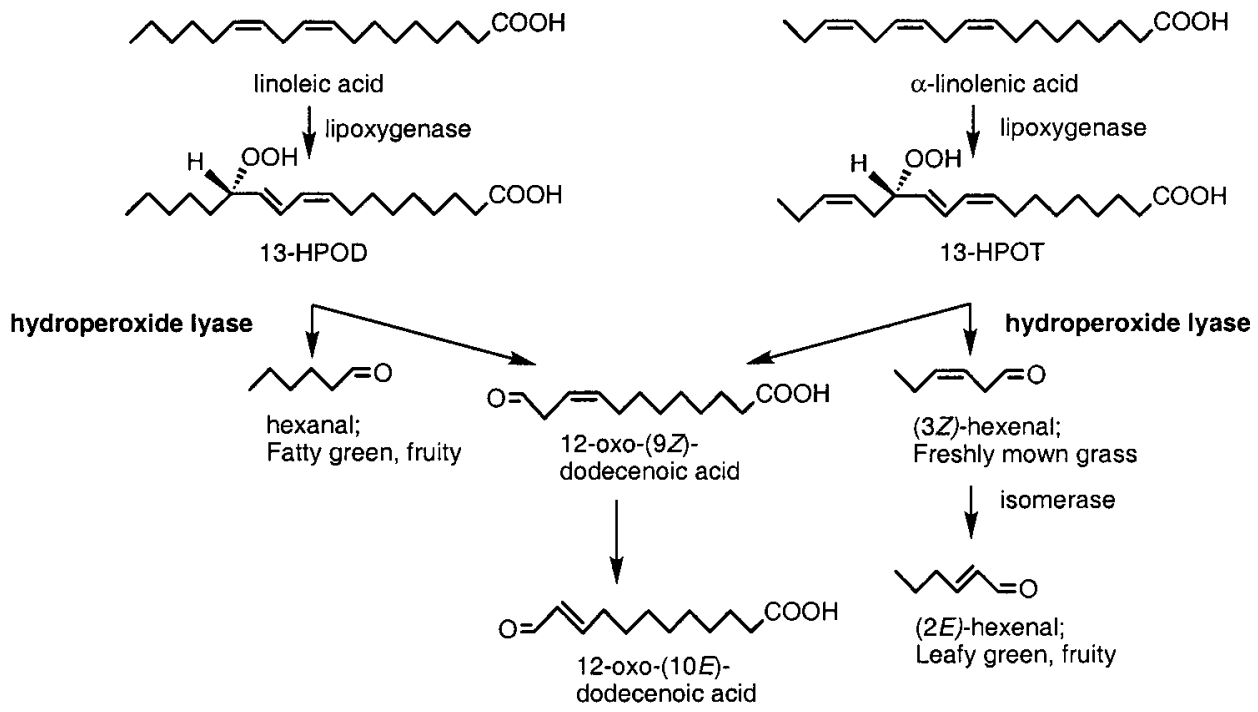

Figure 1. C6-Aldehyde-forming system in plants. C9-Aldehydes are formed similarly from 9-hydroperoxy fatty acids.

recombinant HPL were determined, as well as the kinetic parameters. A versatile biocatalytic process has been developed in which vegetable oils are converted by soybean lipoxygenase and recombinant hydroperoxide lyase to hexanal and (3Z)- or (2E)-hexenal.

\section{MATERIALS AND METHODS}

Expression and Isolation of HPL. Alfalfa HPL was expressed in M15 Escherichia coli cells containing the pQE32 vector (Qiagen) with the $C Y P 74 B 4 v 1$ gene without $\mathrm{N}$-terminal sequence coding for the first 22 amino acids [EMBL Database, accession no. AJ249245 (13)]. For optimal expression, cells were grown at $37{ }^{\circ} \mathrm{C}$ with maximal stirring and $\mathrm{O}_{2}$ flow, in $1.5 \times$ LB medium supplemented with $0.5 \%$ glucose, $1 \times$ minimal medium buffer, $25 \mu \mathrm{g} \mathrm{mL}^{-1}$ kanamycin, and $50 \mu \mathrm{g} \mathrm{mL}^{-1}$ ampicillin. Expression of HPL was induced at an $A_{600}$ of 0.7 by the addition of $1 \mathrm{mM}$ isopropyl $\beta$-D-thiogalactoside (IPTG), and the cells were further grown for $22 \mathrm{~h}$ at $25^{\circ} \mathrm{C}$ to prevent sequestering of HPL in inclusion bodies. Cells were harvested by centrifugation, and the supernatant was discarded. The cell pellets were resuspended in 50 $\mathrm{mM}$ potassium phosphate buffer, $\mathrm{pH} 8$, and sonicated on ice. Triton $\mathrm{X}-100$ from a $10 \%$ solution (w/v) was added to a final concentration of $0.5 \%$ after sonication, and the suspension was recentrifuged. HPL was present in the supernatant. All steps were carried out at $4{ }^{\circ} \mathrm{C}$. For the determination of the initial turnover rates, $K_{\mathrm{m}}$ and $V_{\max }$, HPL was purified by immobilized metal affinity chromatography as described (13).

HPL Activity Measurements. HPL activity was determined in 50 $\mathrm{mM}$ potassium phosphate buffer, $\mathrm{pH} 8.25$, containing $100 \mu \mathrm{M}$ substrate, at $25{ }^{\circ} \mathrm{C}$. The decrease of $A_{234}$ due to cleavage of the substrate was followed spectrophotometrically. One unit of activity (U) corresponds to the amount of enzyme that converts $1 \mu \mathrm{mol}$ of substrate per minute. The maximal turnover number was determined by measuring the substrate conversion at various enzyme/substrate ratios. Apparent $K_{\mathrm{m}}$ and $V_{\max }$ values were determined from the means of six determinations with concentrations of substrate ranging from 5 to $150 \mu \mathrm{M}$. The data were fitted to the standard Michaelis-Menten kinetic equation (Graphpad Prism).

Biocatalytic Processes. Hydrolyzed safflower oil (73\% linoleic acid) was used as linoleic acid source, and hydrolyzed linseed oil (44\% linolenic acid $/ 20 \%$ linoleic acid) was used as linolenic acid source. The reactions were performed in a 7 or $2 \mathrm{~L}$ stirred tank bioreactor $(2$ or $0.5 \mathrm{~L}$ working volumes, respectively), equipped with four baffles and two impellers (Applikon Dependable Instruments, ADI 1035, Schiedam, The Netherlands). The bioreactor was equipped with a thermometer, an oxygen electrode, and a $\mathrm{pH}$ electrode and was controlled by an Applikon Bio Controller ADI 1030. The reaction mixture contained $10 \mathrm{~g} \mathrm{~L}^{-1}$ defatted soybean flour as lipoxygenase source in $50 \mathrm{mM}$ potassium phosphate buffer, $\mathrm{pH} 8.25$, at $20^{\circ} \mathrm{C}$. The $\mathrm{pH}$ was automatically maintained by the addition of $3 \mathrm{M} \mathrm{NaOH}$. Safflower or linseed oil was linearly added over $1 \mathrm{~h}$ to a final concentration of $40 \mathrm{mM}$ linole(n)ic acid. Oxygen saturation was kept at $100 \%$ by the addition of pure oxygen or air. Stirring speed was 400 rpm. When the lipoxygenase reaction was finished and $A_{234}$ did not increase anymore, the oxygen flow was stopped and $115 \mathrm{U}$ of HPL/ mmol of formed 13-hydroperoxylinoleic acid or $400 \mathrm{U} / \mathrm{mmol}$ of 13hydroperoxylinolenic acid was added. The HPL reaction was performed in a closed vessel with a small headspace volume at $10^{\circ} \mathrm{C}$ with slow stirring. If the hydroperoxy fatty acids were extracted from the LOX mixture prior to incubation with HPL, the LOX mixture was acidified with $\mathrm{HCl}$ to $\mathrm{pH}$ 4. The extraction was performed twice with diethyl ether, and the combined ether fractions were frozen to remove water and concentrated by evaporation. In the combined LOX/HPL system, HPL was added to the lipoxygenase reaction mixture in a ratio of 115 $\mathrm{U} / \mathrm{mmol}$ of linoleic acid added.

Product Analysis. The amount of hydroperoxy fatty acids was determined by HPLC (Cosmosil 5C18-AR column, Waters; MeOH/ $\mathrm{H}_{2} \mathrm{O} / \mathrm{THF} / \mathrm{HAc}, 35 / 35 / 30 / 0.1,1 \mathrm{~mL} \mathrm{~min}^{-1}$ ) with detection at $A_{234}$. To quantify the amount of volatiles formed, samples were diluted $1: 1$ with 2-propanol containing $20 \mathrm{mM}$ octanal as internal standard and analyzed by GC-FID (HP-Innowax column, $30 \mathrm{~m} \times 0.32 \mathrm{~mm}, 0.25 \mu \mathrm{m}$ film thickness; temperature gradient, $40-200{ }^{\circ} \mathrm{C}, 10{ }^{\circ} \mathrm{C} \mathrm{min}{ }^{-1}$; injection temperature, $200{ }^{\circ} \mathrm{C}$ ). Nonvolatile products were extracted from the reaction mixture, reduced, methylated, trimethylsilylated, and analyzed by GC-MS as described before (13).

\section{RESULTS}

Optimization of HPL Expression and Isolation Conditions. The influences of expression temperature and medium on the production of active HPL in $E$. coli were determined. The optimal expression temperature appeared to be $25^{\circ} \mathrm{C}$ (Figure 2). At higher temperatures most of the expressed HPL was inactive and probably located in inclusion bodies. Enriched medium resulted in a higher cell density and an increased amount of HPL produced. HPL expression was maximal in the late log-phase and stationary phase. As cells start to die after $24 \mathrm{~h}$, longer expression times will not lead to a higher expression level. For optimal expression $1 \mathrm{mM}$ IPTG had to be added at $A_{600}<0.8$ (Figure 3A). However, also with lower concentrations of IPTG (until $100 \mu \mathrm{M}$ ) a reasonably good expression was obtained. Because IPTG is rather expensive, the optimal amount of IPTG for industrial application may be $<1 \mathrm{mM}$, from an economic point of view. 

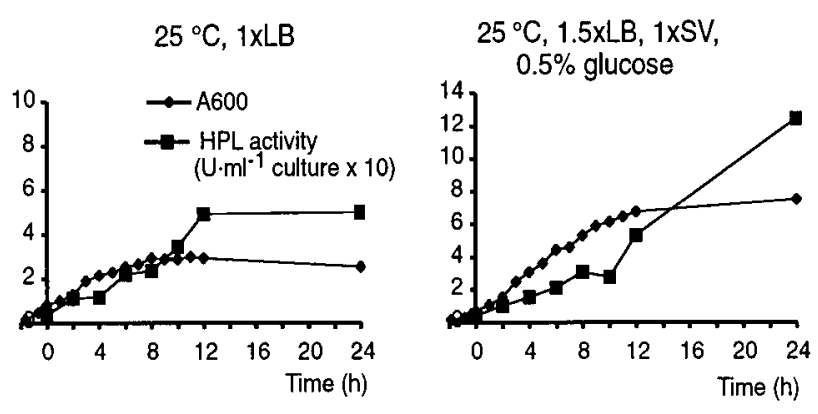

$30^{\circ} \mathrm{C}, 1 \times \mathrm{LB}$
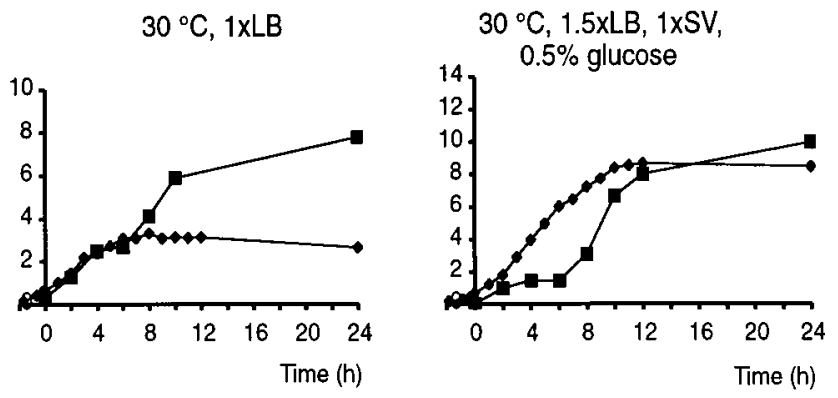

$37^{\circ} \mathrm{C}, 1 \times L B$

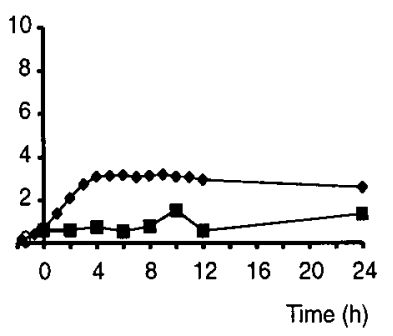

A
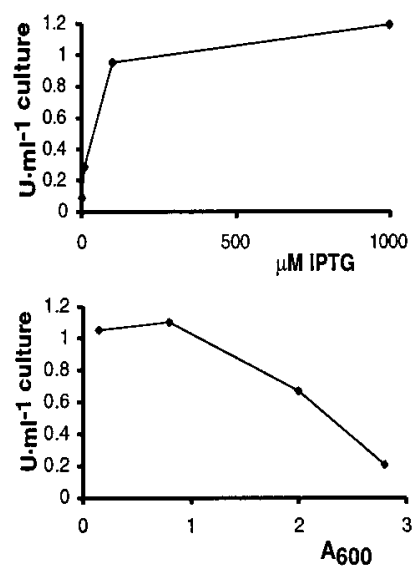

B
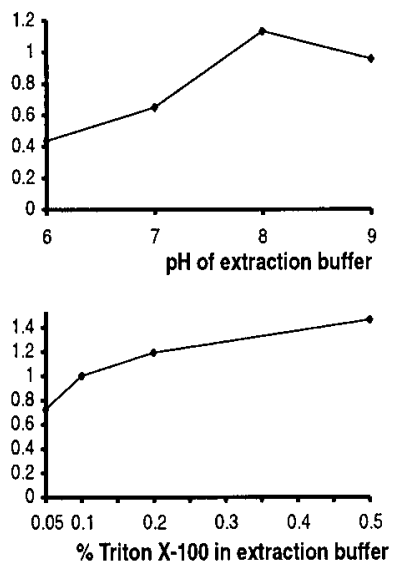

Figure 3. (A) Influence of the amount of IPTG and cell density at the time of induction on the expression of HPL; $(B)$ influence of $\mathrm{pH}$ of the extraction buffer and Triton X-100 concentration on the solubilization of HPL. Small-scale E. coli cultures expressing HPL were grown at $25^{\circ} \mathrm{C}$ in $1 \times$ LB medium. HPL activity was determined with 13-HPOT as substrate.

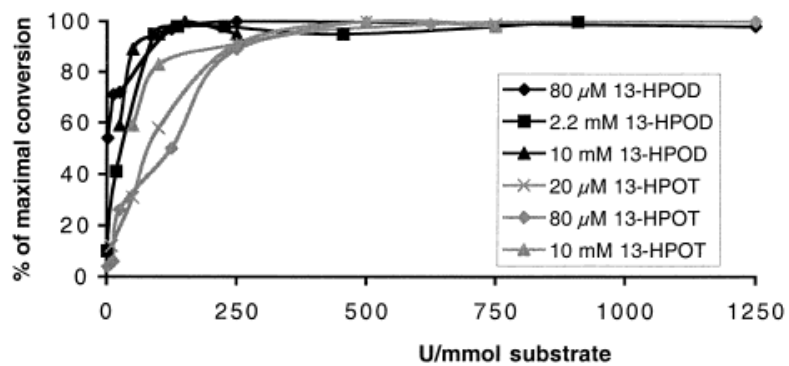

Figure 4. Maximal turnover of substrate by HPL. Different amounts of HPL were incubated with various substrate concentrations. The maximal conversion obtained at each substrate concentration was set to $100 \%$.

reaction temperature from 10 to $50{ }^{\circ} \mathrm{C}$ led to an increase in initial reaction velocity. However, at temperatures $>20^{\circ} \mathrm{C}, \mathrm{HPL}$ was rapidly inactivated, which led to a lower conversion.

The initial turnover rates with $100 \mu \mathrm{M} 13-\mathrm{HPOD}$ or 13-HPOT were 330 or $750 \mathrm{~s}^{-1}$, respectively. To convert $1 \mu \mathrm{mol}$ of 13 HPOD or $1 \mu \mathrm{mol}$ of 13 -HPOT, at least 125 or $400 \mathrm{mU}$ of HPL is needed, respectively (Figure 4). These maximal turnover numbers of recombinant HPL are equal to the turnover numbers of HPL in a crude extract of alfalfa. From these numbers it could be derived that an enzyme molecule can convert $\sim 1.6 \times$ $10^{5}$ molecules of 13 -HPOD or $0.9 \times 10^{5}$ molecules of 13 -HPOT before it is inactivated. Inactivation of HPL was independent of the substrate or product concentration and of the presence of glycerol, Triton X-100, or E. coli membrane fragments. This indicates that inactivation is caused by reaction intermediates. Suicide inactivation was also suggested by Matsui et al., who observed destruction of the heme group during the reaction (15).

Development of a Biocatalytic Process. A process in which the LOX and HPL reactions take place simultaneously has the advantage that there is no product inhibition of LOX, because all product formed by LOX is immediately converted by HPL. As HPL was not inhibited by soybean flour or safflower oil, such a process was worthwhile to study. Although $80 \%$ of the linoleic acid added was converted in this one-step process, the hexanal yield appeared to be quite low (17\%) (Figure 5). Besides low amounts of 9-hydroperoxylinoleic acid and the coproducts 12-oxo-(10E)-dodecenoic acid and 12-oxo-(9Z)dodecenoic acid, no other products were detected, due to the high specificity of recombinant HPL. The addition of oxygen 


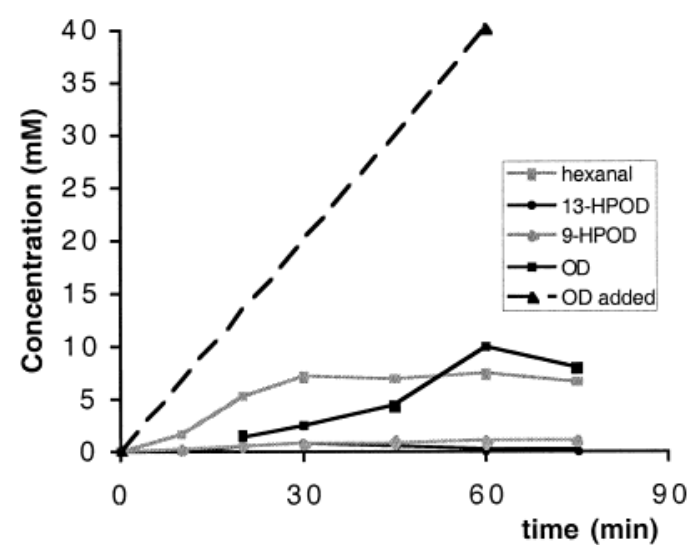

Figure 5. Hexanal formation in a one-step LOX-HPL process. Hydrolyzed safflower oil was linearly added over $1 \mathrm{~h}$ to a concentration of $40 \mathrm{mM}$ OD. HPL was simultaneously added in a ratio of $115 \mathrm{U} / \mathrm{mmol}$ of OD. The reaction mixture contained $10 \mathrm{~g} \mathrm{~L}^{-1}$ soybean flour.

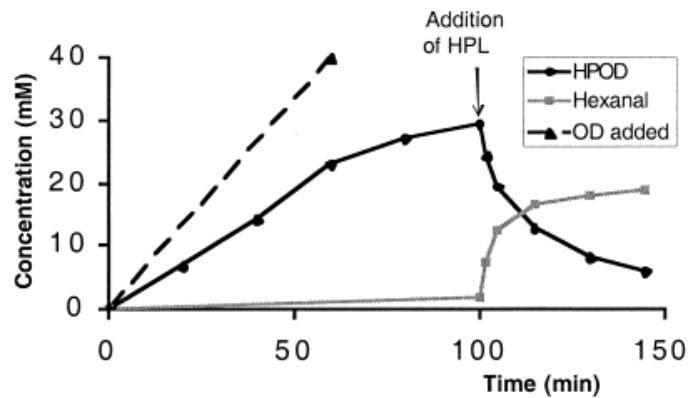

Figure 6. Hexanal formation in a two-step biocatalytic process. Hydrolyzed safflower oil was linearly added over $1 \mathrm{~h}$ to a concentration of $40 \mathrm{mM}$ OD. HPL was added in a ratio of $115 \mathrm{U} / \mathrm{mmol}$ of formed13-HPOD after the LOX reaction was finished and the oxygen flow was stopped.

or air needed for the LOX reaction probably led to evaporation of the volatile aldehydes formed in the HPL reaction. Therefore, a two-step process in which the oxygen flow was stopped before HPL addition was preferred. The yield of the lipoxygenase reaction was again typically $\sim 80 \%$. Subsequently, up to $65 \%$ of the formed 13-hydroperoxylinoleic acids was converted into hexanal, indicating that evaporation was indeed one of the reasons for the low yield of the combined process (Figure 6). The overall yield of the two-step process was 50\%. Another cause of product loss might be the binding of aldehydes or hydroperoxy fatty acids to proteins in the reaction mixture (16, 17). To reduce the protein concentration during the HPL reaction, the hydroperoxy fatty acids were extracted from the LOX mixture prior to addition of HPL. The hexanal yield appeared to be independent of protein and substrate concentrations (ranging from 0.1 to $25 \mathrm{mM} 13-\mathrm{HPOD}$ ), and extraction of hydroperoxylinoleic acid did not lead to higher yields.

Conversion of linseed oil by soybean lipoxygenase gave yields of $\sim 60 \%$. When the LOX mixture was incubated with HPL, (3Z)-hexenal was formed, which slowly isomerized to (2E)-hexenal (Figure 7). The isomerization velocity increased with increasing temperature. Because linseed oil contains both linoleic and linolenic acid, hexanal was formed as well. Due to the preference of HPL for 13-HPOT, hexanal formation was much slower than hexenal formation. If the reaction was stopped after 2 min, a high percentage of (3Z)-hexenal could be obtained ( $~ 80 \%$ of the C6-aldehydes). Although $85 \%$ of 13 -HPOT was converted after $2 \mathrm{~min}$, the hexenal yield was only $43 \%$. The appearance in time of a strong brown-yellow color indicated the formation of Schiff bases with proteins (18). Extraction of

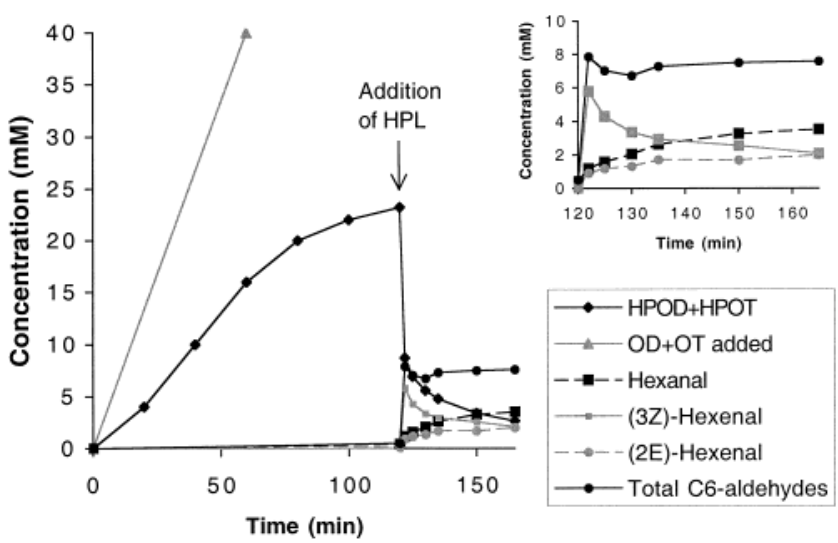

Figure 7. C6-Aldehyde formation in a two-step biocatalytic process with hydrolyzed linseed oil as substrate (linearly added over $1 \mathrm{~h}$ to a concentration of $40 \mathrm{mM} \mathrm{OD}+\mathrm{OT}$ ). HPL was added in ratios of 115 $\mathrm{U} / \mathrm{mmol}$ of formed $13-\mathrm{HPOD}$ and $400 \mathrm{U} / \mathrm{mmol}$ of formed 13-HPOT after the LOX reaction was finished and the oxygen flow was stopped. (Inset) Enlargement of C6-aldehyde formation after addition of HPL.

Table 1. Starting Material Needed per Gram of Produced Aldehyde

\begin{tabular}{lcc}
\hline & hexanal & (3Z)- or (2E)-hexenal \\
\hline safflower or linseed oil & $8.2 \mathrm{~g}$ & $26 \mathrm{~g}$ \\
$\mathrm{~K}_{2} \mathrm{HPO}_{4}$ & $5.1 \mathrm{~g}$ & $15 \mathrm{~g}$ \\
$\mathrm{KH}_{2} \mathrm{PO}_{4}$ & $0.3 \mathrm{~g}$ & $0.9 \mathrm{~g}$ \\
soybean flour & $5 \mathrm{~g}$ & $15 \mathrm{~g}$ \\
E. coli culture expressing HPL & $0.6 \mathrm{~L}$ & $1.6 \mathrm{~L}$ \\
molar conversion & $50 \%$ & $26 \%$ \\
\hline
\end{tabular}

the hydroperoxy fatty acids from the LOX mixture prior to incubation with HPL did not lead to a higher hexenal yield after 2 min. The yield could be increased only by lowering the substrate concentration.

For the isolation of the formed aldehydes from the reaction mixture, the following methods were compared: (1) extraction with organic solvents, (2) steam distillation, and (3) binding to MN-100 (Purolite), a resin that is often used for flavors. Extraction with diethyl ether or ethyl acetate gave very high yields and high purity, because a large fraction of the fatty acids remained in the water phase at the used $\mathrm{pH}$ of 8.25. Separation of the organic layer from the water phase, however, was difficult because of denatured proteins on the interphase. Steam distillation also gave good yields but had to be performed under anaerobic conditions to prevent oxidation of the aldehydes to acids. Furthermore, this method was not suitable for (3Z)hexenal because the high temperature led to isomerization to (2E)-hexenal. Binding to $\mathrm{MN}-100$ and elution with organic solvent gave low yields $(\sim 50 \%)$, because the presence of proteins in the mixture led to strongly decreased binding of the aldehydes to the resin.

\section{DISCUSSION}

The biocatalytic process described here allows rapid, efficient, and cost-effective generation of C6-aldehydes, including (3Z)hexenal. The dioxygenation of linoleic and linolenic acids from hydrolyzed vegetable oils by soybean LOX results in high yields of hydroperoxy fatty acids, as described before $(3-6)$. However, in contrast to previously described processes with plant extracts or cell cultures, the use of recombinant HPL leads to higher aldehyde yields and fewer side products. The total amounts of starting material for the production of $1 \mathrm{~g}$ of hexanal or (3Z)or $(2 E)$-hexenal are shown in Table 1. The HPL reaction can 
easily be scaled up as it is a very simple batch process; no extensive mixing is required, and no foaming occurs.

The process is versatile, as a range of products can be obtained by changing the reaction conditions. Pure hexanal can be produced from safflower oil, and the absence of isomerase activity in recombinant HPL makes it possible to produce (3Z)hexenal from linseed oil. Short reaction times result in a high percentage of (3Z)-hexenal, whereas longer incubations give a mixture of hexanal, (3Z)-hexenal, and (2E)-hexenal. (3Z)Hexenal can rapidly be isomerized to $(2 E)$-hexenal by heating. Using commercial baker's yeast (Saccharomyces cerevisiae), the formed aldehydes can easily be reduced to the corresponding alcohols $(11,19)$.

Although $>90 \%$ of the hydroperoxy fatty acids were converted by HPL, the C6-aldehyde yield was significantly lower. Hexenal seemed to form Schiff bases with proteins, as a strong yellow-brown color appeared in time. Extraction of the hydroperoxy fatty acids from the LOX mixture prior to incubation with HPL did not lead to higher yields, indicating that not only soybean proteins but also E. coli proteins from the HPL mixture are causing product loss. The protein concentration can be minimized by using purified HPL. This is, however, an expensive and time-consuming technique, making it less suitable for industrial purposes (13). Hexanal did not bind to proteins to a large extent, as the hexanal yield was independent of the substrate and protein concentrations. Products formed from C6aldehydes in secondary reactions could not be determined. Polymerization of hydroperoxy fatty acids might be another reason for product loss (20).

\section{ABBREVIATIONS USED}

HPL, hydroperoxide lyase; 13-HPOD, 13-hydroperoxylinoleic acid; 13-HPOT, 13-hydroperoxylinolenic acid; IPTG, isopropyl $\beta$-D-thiogalactoside; LOX, lipoxygenase; OD, linoleic acid, (9Z,$12 Z$ )-octadecadienoic acid; OT, $\alpha$-linolenic acid, $(9 Z, 12 Z, 15 Z)$ octadecatrienoic acid.

\section{ACKNOWLEDGMENT}

We thank M. Franssen and C. Weijers, Wageningen University, The Netherlands, for useful discussions.

\section{LITERATURE CITED}

(1) Major, R. T.; Marchini, P.; Sproston, T. Isolation from Ginkgo biloba L. of an inhibitor of fungus growth. J. Biol. Chem. 1960, 235, 3298-3299.

(2) Zeringue, H. J. Effects of C-6-C-10 alkenals and alkanals on eliciting a defence response in the developing cotton boll. Phytochemistry 1992, 31, 2305-2308.

(3) Elshof, M. B. W.; Janssen, M.; Veldink, G. A.; Vliegenthart, J. F. G. Biocatalytic large-scale production of 13(S)-hydroperoxy$(9 Z, 11 E)$-octadeca-9,11-dienoic acid from hydrolysed safflower oil by a crude soybean-flour extract as lipoxygenase source. Recl. Trav. Chim. Pays-Bas 1996, 115, 499-504.

(4) Iacazio, G.; Langrand, G.; Baratti, J.; Buono, G.; Triantaphylides, C. Preparative, enzymatic synthesis of linoleic acid (13S)hydroperoxide using soybean lipoxygenase. 1. J. Org. Chem. 1990, 55, 1690-1691.

(5) Drouet, P.; Thomas, D.; Legoy, M. D. Production of 13(S)hydroperoxy-9(Z),11(E)-octadecadienoic acid using soybean lipoxygenase 1 in a biphasic octane-water system. Tetrahedron Lett. 1994, 35, 3923-3926.
(6) Fauconnier, M.-L.; Marlier, M. An efficient procedure for the production of fatty acid hydroperoxides from hydrolyzed flax seed oil and soybean lipoxygenase. Biotechnol. Tech. 1996, 10, 839-844.

(7) Berger, R. G.; Kler, A.; Drawert, F. C6-aldehyde formation from linolenic acid in fruit cells cultured in vitro. Plant Cell Tissue Organ Cult. 1987, 8, 147-151.

(8) Chou, S.; Chin, C. Control of the production of cis-3-hexenal, lipid-derived flavor compound, by plant cell culture. In Lipids in Food Flavours; Ho, C.-T., Hartman, T. G., Eds.; ACS Symposium Series 558; American Chemical Society: Washington, DC, 1994; Chapter 19, pp 282-291.

(9) Almosnino, A. M.; Bensoussan, M.; Belin, J. M. Unsaturated fatty acid bioconversion by apple pomace enzyme system. Factors influencing the production of aroma compounds. Food Chem. 1996, 55, 327-332.

(10) Cass, B. J.; Schade, F.; Robinson, C. W.; Thompson, J. E.; Legge, R. L. Production of tomato flavor volatiles from a crude enzyme preparation using a hollow-fiber reactor. Biotechnol. Bioeng. 2000, 67, 372-377.

(11) Whitehead, I. M.; Muller, B. L.; Dean, C. Industrial use of soybean lipoxygenase for the production of natural green note flavor compounds. Cereal Foods World 1995, 40, 193-197.

(12) Rehbock, B.; Gansser, D.; Berger, R. G. Efficient generation of $2 E$-hexenal by a hydroperoxide lyase from mung bean seedlings. Food Chem. 1998, 63, 161-165.

(13) Noordermeer, M. A.; Dijken, A. J. H.v.; Smeekens, S. C. M.; Veldink, G. A.; Vliegenthart, J. F. G. Characterization of three cloned and expressed 13-hydroperoxide lyase isoenzymes from alfalfa with unusual $\mathrm{N}$-terminal sequences and different enzyme kinetics. Eur. J. Biochem. 2000, 267, 2473-2482.

(14) Noordermeer, M. A.; Veldink, G. A.; Vliegenthart, J. F. G. Alfalfa contains substantial 9-hydroperoxide lyase activity and a 3Z:2E-enal isomerase. FEBS Lett. 1999, 443, 201-204.

(15) Matsui, K.; Miyahara, C.; Wilkinson, J.; Hiatt, B.; Knauf, V.; Kajiwara, T. Fatty acid hydroperoxide lyase in tomato fruits: cloning and properties of a recombinant enzyme expressed in Escherichia coli. Biosci. Biotechnol. Biochem. 2000, 64, 11891196.

(16) Jirousova, J.; Davidek, J. Reactions of $n$-hexanal with glycine in model systems. Z. Lebensm. Unters. Forsch. 1975, 157, 269276.

(17) Hidalgo, F. J.; Kinsella, J. E. Changes induced in $\beta$-lactoglobulin B following interactions with linoleic acid 13-hydroperoxide. $J$. Agric. Food Chem. 1989, 37, 860-866.

(18) Davidek, J.; Jirousova, J. Formation of volatile compounds and brown products in the model system $n$-hexanal-glycine. $Z$. Lebensm. Unters. Forsch. 1975, 159, 153-159.

(19) Fauconnier, M.-L.; Mpambara, A.; Delcarte, J.; Jacques, P.; Thonart, P.; Marlier, M. Conversion of green note aldehydes into alcohols by yeast alcohol dehydrogenase. Biotechnol. Lett. 1999, 21, 629-633.

(20) Gardner, H. W. Reactions of hydroperoxides - products of high molecular weight. In Autoxidation of Unsaturated Lipids; Chan, H. W.-S., Ed.; Academic Press: London, U.K., 1987; pp 5194.

Received for review March 1, 2002. Revised manuscript received April 25, 2002. Accepted April 29, 2002. This work was financially supported by Grant IKA94051 from the Department of Economic Affairs, The Netherlands.

JF0202685 